\title{
Competências para o cuidado em saúde de lésbicas, gays, bissexuais, travestis e transexuais: desenvolvimento e validação de instrumento avaliativo'
}

\section{Competency for the health care of lesbians, gays, bisexuals, and transgenders: development and validation of an assessment instrument}

\section{Sávio Marcelino Gomes ${ }^{\mathrm{a}}$}

(D) https://orcid.org/0000-0002-6320-2502

E-mail: savio.gomes.105ळufrn.edu.br

Luiz Roberto Augusto Noro

(D) https://orcid.org/0000-0001-8244-0154

E-mail: luiz_noroळhotmail.com

anniversidade Federal do Rio Grande do Norte. Centro de Ciências da Saúde. Programa de Pós-Graduação em Saúde Coletiva. Natal, RN, Brasil.

\section{Correspondência}

Sávio Marcelino Gomes

Av. Sen. Salgado Filho, 1787. Lagoa Nova, Natal, RN, Brasil. CEP 59056-000.

\section{Resumo}

O estudo busca validar questões avaliativas para compreensão da inserção das temáticas de saúde LGBT na formação universitária na área da saúde. Para isso, foi realizado um estudo de validação de conteúdo em três etapas: construção de modelo lógico, construção de questões avaliativas e análise por especialistas. O modelo lógico foi elaborado a partir de consulta documental à legislação brasileira pertinente, dando origem a dimensões de análise traduzidas em questões avaliativas para, por fim, serem validadas por um painel de 19 especialistas por meio da técnica de consenso de Delfos, em 3 rodadas. Foi analisada a tendência central e dispersão para consolidação das questões, sendo validadas aquelas que obtiveram média $>9$, mediana 10 e desvio-padrão $<1,5$. 0 instrumento validado é composto por 39 itens, em duas dimensões, a saber: Formação de recursos humanos e Concepções, divididas em subdimensões. As subdimensões do primeiro caso são: identidade de gênero, orientação sexual, assistência à saúde e políticas públicas; as do segundo são: concepções individuais, comunitárias e sistêmicas. Este estudo propôs superar os desafios encontrados na literatura, transformando conceitos em categorias analíticas, para que assim possa ser utilizado para fins de pesquisa e/ou autoavaliação de currículos e disciplinas dos diferentes cursos.

Palavras-chave: Recursos Humanos; Gênero e Saúde; Minorias Sexuais e de Gênero; Avaliação. 


\section{Introdução}

This study aims to validate evaluative questions for the integration of LGBT health in undergraduate health training. To this end, a three-stage content validation study was conducted, including: logical model construction, evaluative questions construction, and expert analysis. The logical model was elaborated from a documentary consultation to the relevant Brazilian legislation, resulting in a set of dimensions of analysis that were translated into evaluative questions. These questions were then validated by a panel of 19 experts using the Delphi technique, which builds consensus through three rounds. Questions were consolidated by central tendency and dispersion analysis, and those with mean $>9$, median $=10$, and standard deviation $<1.5$ were validated. The final instrument comprises 39 questions divided into two dimensions: human resources formation, subdivided into sexual identity, sexual orientation, healthcare, and public policies; and Conceptions, including individual, community, and systemic conceptions. This study sought to bridge the gaps on the literature, turning ideas into analytical categories and thus enabling their use for research purposes and/or the evaluation of different courses curricula and disciplines.

Keywords: Human Resources; Gender and Health; Sexual and Gender Minorities; Educational Assessment.
Nas últimas décadas, a incorporação das questões de gênero e sexualidade em estratégias de vigilância em saúde tem possibilitado constatar a magnitude das desigualdades vivenciadas pela população de lésbicas, gays, bissexuais, travestis e transexuais (LGBT), como problemas de saúde mental, enfrentamento de preconceitos, riscos para doenças crônicas, entre outras (Gonzales; HenningSmith, 2017; Grant et al., 2011).

Por conferir parcela de responsabilidade desse quadro aos profissionais de saúde, a American Medical Association (AMA) e a Association of American Medical Colleges (AAMC) recomendam inclusão de tópicos focados em usuários LGBT na educação médica (Greene et al., 2018). As estratégias de implementação dessas recomendações vêm sendo divulgadas, majoritariamente, nos Estados Unidos e Reino Unido, evidenciando resultados promissores quanto à transformação na atuação profissional, à luz de uma diversidade de abordagens, metodologias e ênfases adotadas nos diferentes contextos e programas promotores (Sekoni et al., 2017).

No Brasil, esforços de pesquisadoras e pesquisadores, bem como de movimentos sociais, evidenciam as desigualdades históricas vivenciadas no Sistema Único de Saúde (SUS), como o não atendimento à individualidade desses sujeitos, condutas baseadas na heteronormatividade e consequente afastamento dessa população do serviço. 0 não respeito à identidade de gênero, o assédio sexual, grande número de mortes e violências são muitas vezes justificadas por parte dos profissionais pela não compreensão desses corpos e vivências durante a formação profissional (Brasil, 2018; Gomes et al., 2018).

A Política Nacional de Saúde Integral de LGBT (PNSI-LGBT), estratégia de enfrentamento da desigualdade no SUS e de cuidado adequado à diversidade dos sujeitos, prevê a qualificação profissional para atuação com a diversidade dos territórios (Brasil, 2011). Entretanto, há camadas de complexidades que se colocam como barreiras para a incorporação das abordagens de identidade de gênero e orientação sexual na formação em saúde, como o senso comum, o biologicismo e positivismo (Rufino; Madeiro; Girão, 2013). 
As inserções desses temas feitas na formação em saúde, por sua vez, são incipientes e apresentam diversas abordagens conceituais e metodológicas, delimitando o universo de estudo, em sua maioria, às escolas de medicina, focando em suas atuações particulares. Em função disso, fica inviabilizada a identificação das demandas de outras profissões do campo da saúde e, consequentemente, a produção do conhecimento necessário à reorientação da formação para o SUS.

Diante dessa problemática, este estudo propõe a validação de um instrumento que tem por finalidade identificar a inserção das demandas de saúde LGBT na formação em recursos humanos em saúde, evidenciando as principais potencialidades ou fragilidades dos cursos e/ou instituições na formação de recursos humanos capazes de lidar com a diversidade nos territórios.

\section{Metodologia}

Trata-se de um estudo de validação de conteúdo de questões avaliativas sobre a formação, com abordagem qualitativa em suas duas primeiras etapas: construção do modelo lógico e construção de questões avaliativas; e com abordagem quantitativa relativa à análise dos especialistas. A validação de conteúdo é responsável por iniciar a associação entre conceitos abstratos e critérios mensuráveis, delimitando como cada item representa o fenômeno que se deseja investigar (Rubio et al., 2003).
A pesquisa está de acordo com o preconizado pela Resolução no 466/2012 do Conselho Nacional de Saúde (CNS) quanto aos princípios de sigilo do participante, e foi aprovada pelo Comitê de Ética em Pesquisa (CEP) do Hospital Universitário Onofre Lopes (HUOL) da Universidade Federal do Rio Grande do Norte (UFRN), sob CAAE nº 99895918.o.0ooo.5292.

\section{Construção do modelo lógico}

Levando em conta que as opções técnicas são consideradas também referenciais teóricos, a construção do modelo aqui desenhado emerge da consulta documental aos marcos normativos das políticas públicas nacionais e relatórios de conferências da população LGBT (Brasil, 2004; Brasil, 2008; Brasil, 2009; Brasil, 2011; Brasil, 2013a; Brasil, 2013b; Brasil, 2016b;a,c; Brasil, 2018), à luz das Diretrizes Curriculares Nacionais (DCN) para os cursos de graduação em saúde, na busca da consolidação de uma imagem-objetivo, ou seja, um modelo harmonizado e ordenado, onde sua construção elimina problemas de coerência entre os distintos objetivos, alinhada à legislação oficial brasileira.

Nesse sentido, as categorias emergentes da consulta foram agrupadas de acordo com o objetivo do estudo, de modo a possibilitar a visualização das relações entre elementos essenciais à uma formação comprometida com o cuidado à saúde da população LGBT no SUS (Figura 1).

Figura I - Modelo lógico da formação de recursos humanos para o cuidado integral à população LGBT no SUS.

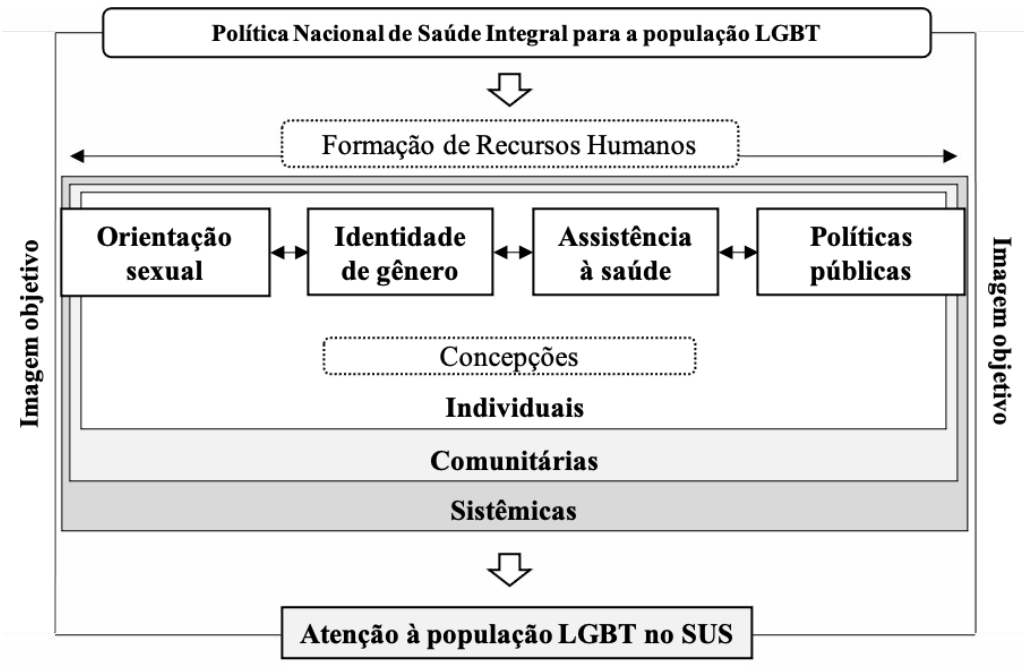




\section{Construção de questões avaliativas}

A partir da construção do modelo lógico foram elaboradas dimensões para a avaliação da formação sobre os componentes essenciais ao cuidado da população LGBT no SUS. Os elementos estruturantes do modelo foram definidos em duas dimensões: (1) Conteúdos formativos e (2) Concepções, a partir das quais foram definidas subdimensões. Para "Conteúdos formativos" foram definidas as subdimensões "orientação sexual”, "identidade de gênero", "assistência à saúde" e "políticas públicas”. Já para "Concepções” foram definidas as subdimensões "individuais", “comunitárias" e "sistêmicas". Para cada critério foram propostas uma ou mais perguntas avaliativas.

\section{Análise por especialistas}

Os marcos normativos brasileiros têm suas origens em diferentes conjunturas, o que acarretou em diferentes abordagens de atuação e de priorização de problemas (Mello; Brito; Maroja, 2012), assim, foi necessária a utilização de uma técnica de consenso que permitisse a consideração de aspectos técnicos sem negligenciar os de ordem ética e política, considerando, ainda, os impactos das experiências do Sul Global, tais como a colonização em formato de exploração e as ditaduras, responsáveis por ampla repressão dos corpos LGBT e produção de violências que se perpetuam até os dias atuais (Connell, 2016).

Foi utilizada para isso a técnica de consenso de Delfos, recomendada para coletar, sistematicamente, juízos e contribuições de especialistas sobre um determinado problema (Valdés; Marín, 2013). Foram seguidos e adaptados os passos preconizados pelo Research guidelines for the Delphi survey technique (Hasson; Keeney; McKenna, 200o), por meio do seguinte percurso metodológico:

1. Seleção de participantes: Buscou-se a seleção de especialistas que pudessem contribuir na consolidação da imagemobjetivo que o estudo se propôs, utilizando, para isso, a técnica de amostragem não probabilística Snowball (Vinuto, 2014), a partir dos seguintes critérios: 1) titulação de mestre ou doutor; 2) atuação em pesquisas empíricas e teóricas no campo do gênero e sexualidade.As sementes, informantes-chave que deram início ao processo de amostragem, foram compostas por pesquisadores participantes de grupos especializados na temática de saúde da população LGBT, em universidades das regiões Nordeste e Sudeste. A elegibilidade das indicações, a partir dos critérios de inclusão na pesquisa foi verificada. Foi solicitado, a cada nova indicação, que indicasse outra pessoa do mesmo grupo ou de um grupo externo ao seu. Quanto ao número de participantes, diante da falta de consenso na literatura, adotou-se a recomendação mínima de Valdés e Marín (2013), de sete especialistas e a recomendação de um máximo ideal de 18. Prevendo as perdas durante o processo, foram convidados 25 especialistas.

2. Coleta de dados: O questionário preliminar, contendo 30 questões construídas a partir das dimensões e subdimensões presentes no modelo lógico, foi enviado para avaliação pelos especialistas. A orientação foi para que os especialistas atribuíssem uma nota para cada item, de o a 10, com a possibilidade também de realizarem comentários qualitativos para reformulação da pergunta e/ou sugestões de exclusão ou adição de novos itens. A metodologia preconiza que esse processo se repita durante três rodadas até a obtenção do consenso.

3. Análise estatística: Foram empregadas, por meio do Software Microsoft Excel, medidas de tendência central e de dispersão, como preconiza Medina et al. (2005), considerando consenso todas as perguntas que apresentassem média >9, desvio-padrão $<1,5$ e mediana 10.

\section{Resultados}

Dos 25 especialistas convidados, 21 retornaram à primeira rodada Delfos, em que foram obtidos consensos em seis itens da matriz e sugestões de 12 acréscimos, além de contribuições qualitativas em todos os itens, que foram reformulados e enviados 
novamente aos especialistas. Na segunda rodada houve perda de 2 especialistas, com validação de mais 30 itens. Uma terceira rodada foi realizada para validação dos itens remanescentes, havendo a perda de um participante e a validação de mais 3 itens. Cada rodada do consenso de Delfos aconteceu no tempo médio de dois meses e a versão final do instrumento é composta de 39 itens.

Os especialistas que fizeram parte do painel de validação tinham formação básica nas áreas de Ciências Sociais, Antropologia, Psicologia, Medicina e Enfermagem, com mestrado e/ou doutorado nas mesmas áreas e também Saúde Coletiva, atuando nos temas: transexualidade e travestilidade em saúde; identidade de gênero e orientação sexual na formação em saúde; demandas da população LGBT; determinação social da saúde da população LGBT e interseccionalidades entre raça, gênero e classe. Atuando, principalmente, nas regiões Nordeste e Sudeste do país. A participação dos especialistas proporcionou, fase a fase, a consolidação de um instrumento que levasse em consideração as experiências latino-americanas, com foco especial no Brasil, contextos com características em comum, como a colonialidade, que repercute nos corpos ainda na contemporaneidade (Connell, 2016).

Na Tabela 1 são expressos os resultados das contribuições e do consenso dos especialistas aos itens considerados essenciais para a formação profissional em saúde, a fim de verificar as reais necessidades de uma formação comprometida com os princípios do SUS e da PNSI-LGBT. Todos os itens para a aplicação do instrumento são acompanhados da questão: "Seu curso de graduação te ofereceu a oportunidade de".

Tabela I - Média, desvio padrão e mediana dos itens relacionados à dimensão "Conteúdos formativos" a partir das subdimensões propostas para consenso dos aspectos analíticos relacionados a formação para o cuidado à população LGBT no SUS

\begin{tabular}{|c|c|c|c|}
\hline Itens & Média & DP & Mediana \\
\hline \multicolumn{4}{|l|}{ Identidade de gênero } \\
\hline Diferenciar identidade de gênero de sexo biológico? & 9,5 & 1,31 & 10 \\
\hline Compreender o conceito de travestilidade? & 9,7 & 1,15 & 10 \\
\hline Compreender o conceito de transexualidade? & 9,7 & 1,15 & 10 \\
\hline Buscar a bibliografia sobre o tema gênero? & 9,0 & 1,30 & 10 \\
\hline Discutir sobre necessidades de saúde de pessoas travestis e transexuais? & 10,0 & 0,01 & 10 \\
\hline Ouvir uma pessoa travesti e/ou transexual falar sobre suas necessidades de saúde? & 9,0 & 1,43 & 10 \\
\hline $\begin{array}{l}\text { Ouvir um profissional de saúde falar sobre a experiência no acolhimento e cuidado à saúde } \\
\text { de pessoas travestis e transexuais? }\end{array}$ & 9,9 & 0,46 & 10 \\
\hline \multicolumn{4}{|l|}{ Orientação sexual } \\
\hline Compreender o conceito de orientação sexual? & 10,0 & 0,01 & 10 \\
\hline Buscar a bibliografia sobre o tema orientação sexual? & 9,4 & 1,12 & 10 \\
\hline $\begin{array}{l}\text { Compreender as diferenças entre as vivências lésbicas, gays e bissexuais nos serviços } \\
\text { de saúde? }\end{array}$ & 9,2 & 1,42 & 10 \\
\hline Discutir sobre necessidades de saúde de pessoas lésbicas, gays e bissexuais? & 9,4 & 1,34 & 10 \\
\hline Ouvir uma mulher lésbica falar sobre necessidades de saúde de mulheres lésbicas? & 9,8 & 0,71 & 10 \\
\hline Ouvir um homem gay falar sobre necessidades de saúde de homens gays? & 9,8 & 0,71 & 10 \\
\hline Ouvir uma pessoa bissexual falar sobre necessidades de saúde de mulheres e homens bissexuais? & 9,6 & 1,12 & 10 \\
\hline Ouvir um profissional de saúde abordar o tema saúde para pessoa lésbica, gay ou bissexual? & 9,3 & 1,27 & 10 \\
\hline
\end{tabular}




\section{Tabela I-Continuação}

Itens

Assistência à saúde

Conhecer as barreiras que a população lésbica enfrenta ao buscar o Sistema Único de Saúde?

Conhecer as barreiras que a população gay enfrenta ao buscar o Sistema Único de Saúde?

Conhecer as barreiras que a população bissexual enfrenta ao buscar o Sistema Único de Saúde?

Conhecer as barreiras que a população travesti e transexual enfrentam ao buscar o Sistema

Único de Saúde?

Compreender como reduzir as principais barreiras que a população lésbica, gay, bissexual, travesti e transexual enfrenta ao buscar os serviços de saúde?

Conhecer sobre o direito ao uso do nome social pelas pessoas travestis e transexuais nos serviços de saúde?

Empregar o nome social no atendimento de pessoas travestis e transexuais?

Identificar práticas de preconceito e discriminação contra pessoas lésbicas, gays,

bissexuais, travestis e transexuais em serviços de saúde?

Como combater o preconceito e discriminação contra pessoas lésbicas, gays, bissexuais, travestis e transexuais em serviços de saúde?

Compreender como proceder ao receber, no serviço de saúde, pessoas lésbicas, gays, bissexuais, travestis ou transexuais que tenham sofrido algum tipo de violência (física,

psicológica, verbal, institucional, intrafamiliar, moral, patrimonial, sexual, simbólica ou outra)?

Compreender o processo transexualizador ofertado pelo sUS?

Compreender como proceder ao receber pessoas travestis e transexuais que busquem por mudanças corporais nos serviços públicos de saúde?

\begin{tabular}{|c|c|c|}
\hline 9,5 & 1,31 & 10 \\
\hline 9,4 & 1,35 & 10 \\
\hline 9,6 & I,0I & 10 \\
\hline 9,6 & 1,30 & 10 \\
\hline 9,8 & 0,54 & 10 \\
\hline 9,9 & 0,32 & 10 \\
\hline 9,4 & 1,34 & 10 \\
\hline 9,9 & 0,23 & 10 \\
\hline 9,0 & 1,34 & 10 \\
\hline 9,7 & $\mathrm{I}, 15$ & 10 \\
\hline 9,7 & 0,78 & 10 \\
\hline 9,7 & 1,15 & 10 \\
\hline
\end{tabular}

$9,7-1,15-10$

\section{Políticas públicas}

Conhecer a Política Nacional de Saúde Integral para a população Lésbica, Gay, Bissexual, Travesti e Transexual (PNSI-LGBT)?

Participar de experiências que contemplassem os objetivos da Política Nacional de Saúde Integral para a população Lésbica, Gay, Bissexual, Travesti e Transexual?

Explorar as políticas e ações em nível estadual e municipal para promoção de saúde da população lésbica, gay, bissexual, travesti e transexual?

Destinado, a priori, à estudantes da área da saúde, essa primeira parte do instrumento caracteriza-se a partir de variáveis qualitativas dicotômicas, a serem respondidas por sim ou não, com o objetivo de identificar o acesso dos estudantes às questões de gênero e sexualidade em sua formação institucional, compreendendo a formação como composta pelas atividades curriculares e extracurriculares.
Para ampliar o referencial, a segunda parte do instrumento visa identificar as opiniões sobre todo o percurso de vida dos estudantes no momento da entrevista, a partir de situações expostas na Tabela 2, em que se expõe também o consenso dos especialistas.

A segunda parte do instrumento caracteriza-se por variáveis qualitativas multinominais, a expressar o nível de concordância em escala (concordo, não concordo e não tenho opinião formada). 
Tabela 2 - Média, desvio padrão e mediana dos itens relacionados à dimensão "Concepções" a partir das subdimensões propostas para consenso dos aspectos analíticos relacionados a formação para o cuidado à população LGBT no SUS

\begin{tabular}{|c|c|c|c|}
\hline Itens & Média & DP & Mediana \\
\hline \multicolumn{4}{|l|}{ Individual } \\
\hline $\begin{array}{l}\text { Pessoas travestis e transexuais são acolhidas no serviço de saúde da mesma forma que } \\
\text { pessoas que não são travestis ou transexuais }\end{array}$ & 9,9 & 0,32 & 10 \\
\hline $\begin{array}{l}\text { A orientação sexual do indivíduo não tem influência no atendimento recebido no } \\
\text { serviço de saúde }\end{array}$ & 9,7 & 0,82 & 10 \\
\hline \multicolumn{4}{|l|}{ Comunitário } \\
\hline $\begin{array}{l}\text { O serviço público de saúde deve oferecer atenção integral e especializada para } \\
\text { modificações corporais em pessoas travestis e transexuais }\end{array}$ & 9,7 & 0,81 & 10 \\
\hline $\begin{array}{l}\text { Identidade de gênero e Orientação sexual devem aparecer nos protocolos de } \\
\text { atendimento nos serviços de saúde }\end{array}$ & 9,9 & 0,23 & 10 \\
\hline $\begin{array}{l}\text { Identidade de gênero e Orientação sexual não devem ser consideradas nas } \\
\text { notificações de violência e mortalidade da população }\end{array}$ & 9,8 & 0,50 & 10 \\
\hline $\begin{array}{l}\text { Os serviços de saúde devem promover eventos com enfoque na saúde de lésbicas, } \\
\text { gays ,bissexuais, travestis e transexuais }\end{array}$ & 10,0 & 0,01 & 10 \\
\hline \multicolumn{4}{|l|}{ Sistêmico } \\
\hline $\begin{array}{l}\text { Não é importante a existência de uma política pública de saúde específica para } \\
\text { lésbicas, gays, bissexuais, travestis e transexuais }\end{array}$ & 9,8 & 0,54 & 10 \\
\hline $\begin{array}{l}\text { A política de saúde do homem deve abordar também homens transexuais, bem como } \\
\text { gays e bissexuais }\end{array}$ & 9,7 & 1,15 & 10 \\
\hline $\begin{array}{l}\text { A política de saúde da mulher deve abordar também mulheres transexuais, travestis, } \\
\text { bem como lésbicas e bissexuais }\end{array}$ & 9,6 & 1,22 & 10 \\
\hline $\begin{array}{l}\text { Não é necessária a inserção da temática Saúde Integral de Lésbicas, Gays, Bissexuais, } \\
\text { Travestis e Transexuais nos cursos de graduação em saúde }\end{array}$ & 9,8 & 0,63 & 10 \\
\hline
\end{tabular}

\section{Discussão}

O instrumento formulado condensa importantes aspectos da determinação social da saúde da população LGBT, que devem ser considerados na formação de profissionais. Considera, para além do plano teórico-conceitual, a necessidade de atuação prática e de exercício do cuidado às populações de gênero e orientação sexual diversas, em diferentes dimensões e níveis de atuação. Assim, se mostra uma importante ferramenta para mensurar as necessidades de qualificação de profissionais de saúde, de modo que a atuação se torne cada vez mais comprometida com os princípios do SUS e a promoção da saúde coletiva.
No período entre 2004 e 2011, uma série de políticas públicas foram implementadas no Brasil, visando a erradicação da discriminação à população LGBT, com destaque ao Programa Brasil sem Homofobia (Brasil, 2004) e a PNSILGBT (Brasil, 2011), esses documentos são importantes ferramentas de orientação das práticas profissionais em saúde, em conjunto com a política nacional de reorientação da formação profissional para o SUS, que objetiva, por sua vez, uma atuação que preze pelos princípios da Reforma Sanitária Brasileira, entre os quais se prevê a equidade e a compreensão da diversidade nos territórios (Dias; Lima; Teixeira, 2013; Mello; Brito; Maroja, 2012). 
No entanto, em relação às políticas de gênero como um todo, a conjuntura pós 2014 é preocupante, especialmente no contexto pós 2018, em que tem início um projeto de governo declaradamente antigênero, com discursos e medidas de controle e opressão de corpos dissidentes, seja por identidade gênero e orientação sexual, seja também por marcadores de raça, etnia e classe. Os direitos passam a ser ameaçados conforme as políticas conquistadas a partir da articulação dos movimentos sociais e governos saem da agenda política, sendo substituídas por práticas e discursos discriminatórios (Nogueira Cunha, 2020).

O deslocamento recente da agenda governamental em relação aos interesses da população LGBT coloca em evidência a necessidade da utilização de documentos normativos na formação dos estudantes. A utilização do nome social, o processo transexualizador e a PNSI-LGBT são apontados no instrumento como documentos essenciais à atuação profissional.

É emergencial explorar os espaços formais de formação de recursos humanos e sua relação com a atuação hegemônica produzida por essas instituições, o potencial transformador de práticas educativas com foco na saúde LGBT foram comprovadas em programas de treinamento nos Estados Unidos, Reino Unido e Quênia. A formação comprometida atua como um recurso político para garantia de uma prática em saúde que considere o corpo em sua integralidade (Sekoni et al., 2017).

Os temas trabalhados em programas de treinamentos para profissionais de saúde sobre a população LGBT são heterogêneos e seus métodos de avaliação, também heterogêneos, inviabilizam uma análise comparada dos currículos. Há registros da avaliação de programas de educação em saúde para profissionais e estudantes da área de saúde desde 1977, em sua maioria focalizados nos cursos de medicina e enfermagem. Os programas apresentam uma ampla variedade de temas, tais como o uso de termos e terminologias, efeitos do estigma e discriminação, sexualidade e disfunção sexual, história sexual e disparidades de saúde. A avaliação desses programas compreenderam uso de técnicas quantitativas e qualitativas de pesquisa (Sekoni et al., 2017).

O LGBT Resource Center da Universidade da Califórnia em San Francisco recomenda diversos temas e métodos para serem trabalhados nas escolas médicas, incluindo temas como risco para doenças crônicas, relações tóxicas, uso de substâncias, saúde adolescente, imagem corporal, transição e cirurgia de redesignação sexual (Hollenbach; Eckstrand; Dreger, 2014).

No entanto, os temas formativos propostos na literatura concentram-se nas experiências norte-americanas, demonstrando a necessidade de se pensar nas especificidades dos corpos LGBTs brasileiros. 0 processo colonial deve ser considerado na análise das necessidades dos corpos do Sul Global. Esses corpos vivenciam experiências atravessadas por apagamentos de suas histórias e de produção de violências, com privação de necessidades básicas de modo distinto do Norte Global (Connell, 2016). As principais conquistas do movimento LGBT brasileiro datam das últimas duas décadas (Melo, 2016). A produção de dados oficiais de violência no sistema de saúde passou a acontecer apenas a partir de 2014 e o Brasil está entre os países mais violentos do mundo para a comunidade LGBT (Pinto et al., 2020). Esse cenário repercute na atuação dos profissionais de saúde no cotidiano dos serviços de saúde, sendo a LGBTfobia² institucional um fator agravante para a determinação social do adoecimento em pessoas LGBT (Gomes et al., 2018).

Temas consensuais entre os participantes da pesquisa traduzem as demandas contemporâneas e emergentes desses processos. Considerar a travestilidade no Brasil é essencial, pois são agentes importantes na luta pelos direitos à saúde da comunidade. Assim como também, em conjunto a transexuais, possuem os maiores índices de vulnerabilidade social (Nascimento; Sousa; Barros, 2019). Do mesmo modo, a prevalência de violências no Brasil impulsionou a inserção do tema no

2 Utilizamos o termo LGBTfobia em substituição ao termo Homofobia, que apesar de ser amplamente conhecido, limita-se à opressão vivenciada por orientação sexual. Sua substituição no texto se justifica pela consideração também, no sentido do texto, de menção à opressão vivenciada pela identidade de gênero. 
instrumento, sendo de fundamental importância que o profissional compreenda aspectos como a vigilância de violências, as notificações compulsórias e quais os pontos da Rede de Atenção à Saúde podem ser acionados para o cuidado integral de pessoas LGBT (Pinto et al., 2020).

Nesse instrumento as concepções dos estudantes compõem, com os tópicos formativos, uma dimensão de análise. As concepções devem também ser exploradas por sua relação com o imaginário social influenciado pelas agendas hegemônicas. Assim, será possível compreender o impacto fenomenológico da formação no modo de pensar e no alinhamento dos estudantes e profissionais com as políticas implementadas (Costa et al., 2017).

Os instrumentos utilizados para essas avaliações ainda são incipientes (Aslan; Şahin; Emiroğlu, 2019; Greene et al., 2018; Parameshwaran et al., 2016) e no Brasil são realizadas majoritariamente por meio de pesquisas qualitativas (Raimondi et al., 2019). A técnica de consenso de Delfos empregada neste estudo foi, portanto, de fundamental importância para a consolidação de um instrumento padronizado, capaz de reunir os principais conceitos de natureza político-social em categorias de análise, a partir do acesso a estudiosos geograficamente dispersos de modo simultâneo (Valdés; Marín, 2013).

Outra situação-limite que se insere nas investigações sobre a formação é sua concentração nas escolas médicas, sendo os principais objetos dos diversos estudos no Brasil e no mundo (Raimondi et al., 2019; Sekoni et al., 2017), situação que este instrumento tem como premissa superar, propondo critérios transversais às diversas formações no campo da saúde que possua inserção no SUS.

O instrumento, como uma ferramenta analítica, buscou sintetizar as principais questões em torno da determinação social da saúde de pessoas LGBT, sem, no entanto, tornar-se extenso e, por conseguinte, de difícil aplicação para diferentes contextos. Assim, não menciona os corpos LGBT de diferentes raças, etnias ou pessoas com deficiência, mas consideramos a importância de que esses fatores sejam aprofundados nas discussões advindas de sua utilização.

\section{Considerações finais}

É urgente a produção de dados sobre como a PNSI-LGBT vem sendo implementada, com especial atenção às instituições de formação de recursos humanos destinados ao SUS, onde a população LGBT historicamente e constantemente é afastada por práticas binárias e heteronormativas.

Este estudo propõe um caminho para investigações desse cunho, superando os desafios apresentados nos estudos de natureza semelhante, ao apresentar, por consenso, a consolidação de conceitos em categorias analíticas essenciais para a formação básica de profissionais de saúde, inerentes à superação da LGBTfobia, emergente nos territórios e instituições que se inserem no SUS.

Sua utilização foi vislumbrada para futuras pesquisas diagnósticas, bem como para autoavaliações de cursos na área de saúde, propondo uma reflexão sobre temas transversais à formação e à inclusão de corpos dissidentes e historicamente ininteligíveis nos espaços de construção de conhecimento.

Torna-se importante, também, como mecanismo de resistência, ao considerar o atual contexto político-social vivenciado, em que as políticas de equidade sofrem especial ameaça por uma onda conservadora que atinge todos os âmbitos da sociedade. Estratégias de consolidação dos princípios do SUS nos territórios e de combate contra os ataques às populações mais vulneráveis devem ser cotidianamente fortalecidas.

\section{Referências}

ASLAN, F.; ŞAHIN, N. E.; EMIROĞLU, O. N. Turkish nurse educators knowledge regarding LGBT health and their level of homophobia: a descriptive-cross sectional study. Nurse Education Today. Amsterdam, n. 76, p. 216-221, 2019. DOI: 10.1016/j. nedt.2019.02.014.

BRASIL. MINISTÉRIO DA SAÚDE. Brasil sem homofobia: programa de combate à violência e à discriminação contra GLTB e de promoção da cidadania homossexual. Brasília, DF, 2004. Disponível em: <https://bit.ly/2Yo3ASk>.

Acesso em: 29 set. 2021. 
BRASIL. MINISTÉRIO DA SAÚDE. Política nacional de saúde integral de lésbicas, gays, bissexuais, travestis e transexuais. Brasília, DF, 2011.

BRASIL. MINISTÉRIO DA SAÚDE. Portaria $\mathrm{n}^{0} 2.803$, de 19 de novembro de 2013. Redefine e amplia o processo transexualizador no Sistema Único de Saúde (SUS). Diário Oficial da União, seção 1, Brasília, DF, 20 nov. 2013a.. p. 25. Disponível em: <https://bit.ly/3ASZlXf>. Acesso em: 28 set. 2021.

BRASIL. MINISTÉRIO DOS DIREITOS HUMANOS. Violência LGBTFóbica no Brasil: dados da violência. Brasília, DF, 2018. Disponível em: <https://bit.ly/3F4Sywo>. Acesso em: 29 set. 2021.

BRASIL. SECRETARIA ESPECIAL DOS DIREITOS HUMANOS. CONFERÊNCIA NACIONAL LGBT, 1., 2008, Brasília, DF. Anais [...]. Brasília, DF, 2008. <Disponível em: https://bit.ly/3kQ7175>. Acesso em: 29 set. 2021.

BRASIL. SECRETARIA ESPECIAL DOS DIREITOS HUMANOS. CONFERÊNCIA NACIONAL LGBT, 2., 2016, Brasília, DF. Anais [...]. Brasília, DF, 2016a. Disponível em: 〈https://bit.ly/2Y5a5nA>. Acesso em: 29 set. 2021.

\section{BRASIL. SECRETARIA ESPECIAL DOS} DIREITOS HUMANOS. Plano Nacional de Promoção da Cidadania e Direitos Humanos de LGBT. Brasília, DF, 2009. Disponível em: <https://bit.ly/2ZC26OZ>. Acesso em 29 set. 2021. BRASIL. SECRETARIA ESPECIAL DOS DIREITOS HUMANOS. Relatório final da 30 conferência nacional de políticas públicas direitos humanos de direitos humanos de lésbicas, gays, bissexuais, travestis e transexuais. Brasília, DF, 2016c. Disponível em: <https://bit.ly/3oggXcb>. Acesso em: 29 set. 2021.

BRASIL. SECRETARIA ESPECIAL DOS DIREITOS HUMANOS. Relatório sobre violência homofóbica no Brasil: ano de 2012. Brasília, DF, 2013b. Disponível em: <https://bit.ly/3B5NPb6>. Acesso em: 29 set. 2021.

BRASIL. SECRETARIA ESPECIAL DOS DIREITOS HUMANOS. Relatório sobre violência homofóbica no Brasil: ano de
2013. Brasília, DF. 2016b. Disponível em: <https://bit.ly/3F6y1Yq>. Acesso em: 29 set. 2021. CONNELL, R. Gênero em termos reais. São Paulo: NVersos, 2016. p. 272.

COSTA, L. D. Da et al. Competência Cultural e Atenção à Saúde da população de lésbicas, gays, bissexuais travestis e transexuais (LGBT). Tempus Actas de Saúde Coletiva, Brasília, DF, v. 11, n. 1, p. 105, 2017. DOI: 10.18569/tempus.v11i1.2314

DIAS, H. S.; LIMA, L. D. De; TEIXEIRA, M. A trajetória da política nacional de reorientação da formação profissional em saúde no SUS. Ciência \& Saúde Coletiva, Rio de Janeiro, v. 18, n. 6, p. 1613-1624, 2013.DOI: 10.159o/S141381232013000600013

GOMES, S. M. et al. O SUS fora do armário: concepções de gestores municipais de saúde sobre a população LGBT. Saúde e Sociedade, São Paulo, v. 27, n. 4, p. 1120-1133, 2018. DOI: 10.1590/So10412902018180393

GONZALES, G.; HENNINGSMITH, C. Health disparities by sexual orientatioin: results and implications from the behavioral risk fator surveillance system. Community Health, [S. l.], v. 42, n. 6, p. 1163-1172, 2017. DOI: 10.1007/s1090oo17-0366-z

GRANT, J. M. et al. Injustice at every turn: a report of the national transgender discrimination survey. Washington, DC: NCTE; National Gay and Lesbian Task Force, 2011. Disponível em: <https://bit.ly/3igpF6o>. Acesso em: 29 set. 2021.

GREENE, M. Z. et al. Comparing medical, dental, and nursing students' preparedness to address lesbian, gay, bisexual, transgender, and queer health. Plos One, [S. l.], v. 13, n. 9, p. 1-16, 2018. DOI: 10.1371/journal.pone.0204104

HASSON, F.; KEENEY, S.; MCKENNA, H. Research guidelines for the Delphi survey technique. Journal of Advanced Nursing, v. 32, n. 4, p. 1008-1015, 200o. Disponível em: <https://bit.ly/39QWFxv>. Acesso em: 29 set. 2021. HOLLENBACH, A.; ECKSTRAND, K.; DREGER, A. Implementing curricular and institutional 
climate changes to improve health care for individuals who are LGBT, gender nonconforming, or born with DSD: a resource for medical educators. Washington, DC: AAMC, 2014.

MEDINA, M. G. et al. Uso de modelos teóricos na avaliação em saúde: aspectos conceituais e operacionais. In: HARTZ, Z. M. A.; SILVA, L. M. V. (Org.). Avaliação em saúde: dos modelos teóricos à prática na avaliação de programas e sistemas de saúde. Salvador, Rio de Janeiro: EDUFBA, Fiocruz, 2005. p. 275. DOI: $10.7476 / 9788575415160$.

MELLO, L.; BRITO, W.; MAROJA, D. Políticas públicas para a população LGBT no Brasil: notas sobre alcances e possibilidades. Cadernos pagu, Campinas, v. 39, p. 403-429, 2012. DOI: 10.1590/So104-83332012000200014.

MELO, I. F. Breves notas históricas sobre sexualidades e identidades de gênero periféricas: o alvorecer do movimento LGBT no mundo. Bagoas, Natal, v. 10, n. 14, p. 205-232, 2016. Disponível em: <https://bit.ly/3F2XH89>. Acesso em: 29 set. 2021.

NASCIMENTO, H. M.; SOUSA, J. A.; BARROS, C. R. S. $O$ atendimento em saúde a travestis e transexuais: revisão sistemática de literatura (2008-2017). Revista Brasileira de Estudos da Homocultura, Redenção, v. 1, n. 4, p. 40-58, 2019. Disponível em: <https://bit.ly/3oiNsgH>. Acesso em: 29 set. 2021.

NOGUEIRA CUNHA, L. L. La antipolítica de género en el gobierno de Bolsonaro y sus dinámicas de violencia. Revista de Estudios Brasileños. Salamanca, v. 7, n. 14, p. 49, 2020. DOI: 10.14201/ reb20207144961

PARAMESHWARAN, V. et al. Is the lack of specific lesbian, gay, bisexual, transgender and queer/ questioning (LGBTQ) healthcare education in medical school a cause for concern? Evidence from a survey of knowledge and practice amongst UK medical students. Journal of Homosexuality. [S. l.], v. 64, n. 3, p. 367-381, 2017. DOI: 10.108o/o0918369.2016.1190218

PINTO, I. V. et al. Perfil das notificações de violências em lésbicas, gays, bissexuais, travestis e transexuais registradas no Sistema de Informação de Agravos de Notificação,
Brasil, 2015 a 2017. Revista Brasileira de Epidemiologia. São Paulo, v. 23, nº suppl 1, 2020. DOI: 10.1590/1980-549720200oo6.supl.1

RAIMONDI, G. A. et al. Ensinoaprendizagem de gênero e sexualidade em um curso de medicina no Brasil: promovendo o cuidado integral em saúde e os direitos humanos. Revista Brasileira de Educação Médica, [S. l.], v. 43, n. 2, p. 130-142, 2019. DOI: 10.159o/1981-52712015V43n2RB20180125

RUBIO, D. M. et al. Objectifying content validity: Conducting a content validity study in social work research. Social Work Research, [S. l.], v. 27, n. 2, p. 94-104, 2003. DOI: 10.1093/swr/27.2.94

RUFINO, A. C.; MADEIRO, A. P.; GIRÃO, M. J. B. C. O Ensino da sexualidade nos cursos médicos: a percepção de estudantes do Piauí. Revista Brasileira de Educação Médica, [S. l.], v. 37, n. 2, p. 178-185, 2013. Disponível em: <https://bit.ly/2WmNbXK>. Acesso em: 29 set. 2021.

SEKONI, A. O. et al. The effects of educational curricula and training on LGBT-specific health issues for healthcare students and professionals: a mixed-method systematic review. Journal of the International AIDS Society, [S. l.], v. 20, n. 1, 21624, 2017. DOI: 10.7448/IAS.20.1.21624

VALDÉS, M. G.; MARÍN, M. S. El método Delphi para la consulta a expertos en la investigación científica. Revista Cubana de Información en Ciencias de la Salud, [S. l.], v. 39, n. 2, p. 253-267, 2013. Disponível em: <https://bit.ly/3F1X7Yc>. Acesso em: 29 set. 2021.

VINUTO, J. A amostragem em bola de neve na pesquisa qualitativa: um debate em aberto. Temáticas, [S. l.], v. 22, n. 44, p. 201-220, 2014. DOI: 10.20396/tematicas.v22i44.10977

\section{Contribuição dos autores}

Comes e Noro foram igualmente responsáveis pela concepção, execução, análise, sistematização dos resultados, redação e revisão final do manuscrito.

Recebido: 10/10/2019

Reapresentado: 01/07/2021

Aprovado: 27/09/2021 